Article

\title{
Muscle Enriched Lamin Interacting Protein (Mlip) Binds Chromatin and Is Required for Myoblast Differentiation
}

\author{
Elmira Ahmady ${ }^{1,2}$, Alexandre Blais ${ }^{1,3,4}$ and Patrick G. Burgon $5, *$ (D) \\ 1 Department of Biochemistry, Microbiology, and Immunology, University of Ottawa, Ottawa, \\ ON K1H 8M5, Canada; elmiraahmady25@gmail.com (E.A.); alexandre.blais@uottawa.ca (A.B.) \\ 2 Molecular Signaling Laboratory, University of Ottawa Heart Institute, Ottawa, ON K1Y 4W7, Canada \\ 3 Ottawa Institute of Systems Biology, University of Ottawa, Ottawa, ON K1H 8M5, Canada \\ 4 University of Ottawa Centre for Infection, Immunity and Inflammation (CI3), Ottawa, ON K1H 8M5, Canada \\ 5 Department of Chemistry and Earth Sciences, College of Arts and Sciences, Qatar University, \\ Doha 999043, Qatar \\ * Correspondence: patrick.burgon@qu.edu.qa
}

Citation: Ahmady, E.; Blais, A.;

Burgon, P.G. Muscle Enriched Lamin Interacting Protein (Mlip) Binds Chromatin and Is Required for Myoblast Differentiation. Cells 2021, 10, 615. https://doi.org/10.3390/ cells10030615

Academic Editor: Thomas Dechat

Received: 18 December 2020

Accepted: 8 March 2021

Published: 10 March 2021

Publisher's Note: MDPI stays neutral with regard to jurisdictional claims in published maps and institutional affiliations.

Copyright: (c) 2021 by the authors. Licensee MDPI, Basel, Switzerland. This article is an open access article distributed under the terms and conditions of the Creative Commons Attribution (CC BY) license (https:// creativecommons.org/licenses/by/ $4.0 /)$.

\begin{abstract}
Muscle-enriched A-type lamin-interacting protein (Mlip) is a recently discovered Amniota gene that encodes proteins of unknown biological function. Here we report Mlip's direct interaction with chromatin, and it may function as a transcriptional co-factor. Chromatin immunoprecipitations with microarray analysis demonstrated a propensity for Mlip to associate with genomic regions in close proximity to genes that control tissue-specific differentiation. Gel mobility shift assays confirmed that Mlip protein complexes with genomic DNA. Blocking Mlip expression in C2C12 myoblasts downregulates myogenic regulatory factors (MyoD and $\mathrm{MyoG}$ ) and subsequently significantly inhibits myogenic differentiation and the formation of myotubes. Collectively our data demonstrate that Mlip is required for $\mathrm{C} 2 \mathrm{C} 12$ myoblast differentiation into myotubes. Mlip may exert this role as a transcriptional regulator of a myogenic program that is unique to amniotes.
\end{abstract}

Keywords: Mlip; ChIP; skeletal muscle

\section{Introduction}

A-type lamins have been implicated in the maintenance of cellular commitment and differentiation. Mutations in the A-type lamin gene ( $L M N A)$ manifest as tissue-specific degenerative diseases (laminopathies) with varied clinical symptoms that include dilated cardiomyopathy, atherosclerosis, muscular dystrophy, lipodystrophy, neuropathy and progeria [1-4]. The molecular mechanisms that link mutations in LMNA with different human diseases are poorly understood at this time. The recent discovery of a unique muscle-enriched A-type lamin-interacting protein (Mlip) [5] may provide valuable new insight into how primary genetic defects in $L M N A$, a ubiquitously expressed nuclear protein, translates to tissue-specific diseases.

Mlip is expressed ubiquitously and most abundantly in heart, skeletal and smooth muscle. The Mlip gene encodes at least seven, alternatively spliced, A-type lamin-interacting factors that possess a unique primary amino-acid sequence not found in other proteins $[5,6]$. Mlip is observed only in amniotes' genomes as a single copy gene with no evidence of duplication [5], suggesting that there may be no functional redundancy. In addition, the type of Mlip isoforms expressed differs between each of the tissues, with the heart being the most heterogeneous in Mlip isoform expression [5,6]. Down-regulation of lamin A/C expression by shRNA results in Mlip's up-regulation and its mislocalization from the nuclear envelope to the nucleoplasm/cytoplasm [5]. Recently, Mlip was shown to interact with Islet1 (Isl1), an essential transcription factor for cardiac progenitor cell specification $[7,8]$, and blocks agonist-induced hypertrophy of cardiomyocytes in culture [9]; Mlip plays a critical role in the maintenance of cardiac homeostasis and protects the heart against pathophysiological 
stresses $[10,11]$ and for normal myonuclear positioning in skeletal muscle [6,12]. Mlip's precise biological role remains to be defined.

Here we report the functional characterization of Mlip as a chromatin-binding protein with transcriptional co-factor activity. Mlip interacts with chromatin in close proximity to many genes that are functionally associated with tissue development and required for the normal myoblast fusion process during differentiation. The findings presented in the current study provide the first insight into the biological function of Mlip.

\section{Materials and Methods}

\subsection{Cell Culture}

The mouse $\mathrm{C} 2 \mathrm{C} 12$ myoblast cell line (American Type culture collection ATCC no. CRL-1772) was cultured at $37^{\circ} \mathrm{C}$ in an atmosphere of $10 \% \mathrm{CO}_{2}$ in growth medium consisting of Dulbecco's modified Eagle's medium (DMEM) supplemented with $20 \%$ fetal bovine serum (FBS), $1 \% v / v$ penicillin-streptomycin and $2 \mathrm{mM}$ L-glutamine. To induce myogenic differentiation, cells with $60-70 \%$ confluency were subjected to differentiation by switching growth medium to differentiation medium (DMEM $+2 \%$ horse serum). For immunofluorescence, staining cells were grown on sterile coverslips coated with gelatin in 12-well cell culture treated dishes.

The mouse HL1 cell line was cultured at $37{ }^{\circ} \mathrm{C}$ in an atmosphere of $5 \% \mathrm{CO}_{2}$, in Claycomb medium (Sigma, Saint louis, MO, USA) supplemented with $10 \%$ fetal bovine serum, $1 \% v / v$ penicillin-streptomycin and $2 \mathrm{mM}$ L-glutamine. The HEK293 cell line was cultured at $37^{\circ} \mathrm{C}$ in an atmosphere of $5 \% \mathrm{CO}_{2}$, in DMEM medium (Thermofisher, Waltham, MA, USA), supplemented with $10 \%$ fetal bovine serum, $1 \% v / v$ penicillin-streptomycin and 2 mM L-glutamine.

\subsection{Whole Cell Extract Preparation for Immunoprecipitation and Western Blotting}

Proteins were extracted using lysis buffer containing: $50 \mathrm{mM}$ Tris- $\mathrm{HCl}[\mathrm{pH}=8.0]$, $200 \mathrm{mM} \mathrm{NaCl}, 20 \mathrm{mM} \mathrm{NaF}, 20 \mathrm{mM} \beta$-glycerolphosphate, $0.5 \% \mathrm{NP}-40,0.1 \mathrm{mM} \mathrm{Na}_{3} \mathrm{Vo}_{4}, 1 \mathrm{mM}$ dithiothreitol, $1 \times$ Protease inhibitor cocktail (Roche, 1 tablet $/ 7.0 \mathrm{~mL}$ ), and phosphatase inhibitor cocktails (Sigma, $0.1 \mathrm{~mL} / 7.0 \mathrm{~mL}$ ). Cells were scraped from dishes and lysates pipetted into $1.5 \mathrm{~mL}$ centrifuge tubes. Lysates were incubated on ice for $20 \mathrm{~min}$, and cleared by centrifugation at $10,000 \times \mathrm{g}$ for $10 \mathrm{~min}$ at $4{ }^{\circ} \mathrm{C}$. Supernatants collected and protein concentrations were determined using the Bradford protein assay (Biorad). Proteins were resolved on a 15\% $0.75 \mathrm{~mm}$ thick SDS-TrisGlycine-Polyacrylamide gel (200 V) and transferred to PVDF membranes (Millipore) for $1 \mathrm{~h}$ at $100 \mathrm{~V}$ at $4{ }^{\circ} \mathrm{C}$. Membranes were blocked in $5 \%$ nonfat milk dissolved in Tris-buffered saline $+0.05 \%$ Tween 20 . Mlip mediated immunoprecipitations were performed as described previously [5].

For Western blot analysis, Mlip antibodies were raised in rabbits against a synthetic peptide N-LRKDEEVYEPNPFSKYL-C (21st Century Biochemicals) [5]. The following primary antibody concentrations were used: Mlip, 1:50,000 of rabbit antibody, Myogenin mouse monoclonal IgG 1:1000 (Clone F5D sc12732; Santa Cruz Biotechnology, Dallas, TX, USA) and anti-alpha-Tubulin mouse monoclonal 1:5000 (T9026, Sigma). MyoD mouse monoclonal IgG 1:500 (Clone 5.8A, sc32758; Santa Cruz Biotechnology) as well as Six1 and Six4 rabbit polyclonal 1:1000 [13].

Membranes were then incubated with HRP-conjugated anti-rabbit or anti-mouse IgG secondary antibody (Santa Cruz Biotechnology). Immunoblot signals were detected with a SuperSignal West Pico Chemiluminescent Kit (Thermo Scientific, Waltham, MA, USA) and visualized on X-ray film (Sigma). 


\subsection{Immunofluorescence}

C2C12 cells were grown on gelatin-coated coverslips in 12-well cell culture treated plates (Fisher Scientific, Waltham, MA, USA). C2C12 cells were differentiated using differentiation medium and fixed at appropriate time points using pre-cooled methanol for $20 \mathrm{~min}$ on ice and were washed carefully twice with phosphate-buffered saline (PBS). To reduce nonspecific binding, cells were blocked with PBS supplemented with $5 \%$ fetal bovine serum and $0.1 \% \mathrm{NP}-40$ in PBS for $30 \mathrm{~min}$ at room temperature with gentle shaking and were then incubated with anti-Mlip (1:1000), anti-MHC antibody (1:500) (Developmental Studies Hybridoma Bank) in 1.5\% FBS/PBS for $1 \mathrm{~h}$ at room temperature followed by fluorescentconjugated secondary antibody Regulus Red 594 anti-mouse IgG (LP Bio). Nuclei were stained with DAPI $(1 \mu \mathrm{g} / \mathrm{mL})$ and mounted with fluorescent mounting medium (Dako). Images were then visualized using epifluorescent microscopy (Carl Zeiss Axio Imager 2) using objective magnification: $10 \times$, numerical aperture: 0.25 , Plan-Apochromat or $20 \mathrm{X}$, numerical aperture: 0.8, Plan-Apochromat. The Illuminator HBO 100 for fluorescence applications was used as a light source. The following Zeiss filter sets were available (46HE; $38 \mathrm{HE}$; $43 \mathrm{HE}$; 47HE; and 25HE) and images were captured with a ZEISS Axiocam 506 color and Axio cam HRM using the Zeiss Zen 2.3 software.

\subsection{Chromatin Immunoprecipitation and ChIP-on-Chip Studies}

$\mathrm{C}_{2} \mathrm{C}_{12}$ myoblast cells were grown in $5 \times 150 \mathrm{~mm}$ cell culture dishes $\left(50 \times 10^{6}\right.$ cells $)$ and subjected to chromatin immunoprecipitation according to manufacturer's protocol (cell signaling). Chromatin was sheared into approximately 5 nucleosomes. Chromatin (250 ug) was immunoprecipitated with a specific Mlip antibody (1:1000), Histone H3 (positive control) and normal rabbit IgG (negative control). DNA linker's oJW102: 5'-GCG GTG ACC CGG GAG ATC TGA ATT C-3' and oJW103: $5^{\prime}$-GAA TTC AGA TC-3' were ligated to recover Mlip ChIP DNA according to the method described by Agilent Mammalian ChIP-on-chip protocol (Agilent). DNA amplification was carried out as follows: $95^{\circ} \mathrm{C}$ for $5 \mathrm{~min}$, followed by 34 cycles in 3 steps: $95^{\circ} \mathrm{C}$ for $30 \mathrm{~s}, 60^{\circ} \mathrm{C}$ for $30 \mathrm{~s}$, and $72{ }^{\circ} \mathrm{C}$ for $30 \mathrm{~s}$ and $72{ }^{\circ} \mathrm{C}$ for $5 \mathrm{~min}$ using Taq DNA polymerase (NEB). PCR products were cleaned using QiAquik PCR purification kit columns (Qiagen). For ChIP DNA sequencing, $4.0 \mu \mathrm{L}$ of Mlip linkered PCR product was TOPO-TA cloned into pCRII-TOPO (Invitrogen) and transformed into bacteria.

ChIP-on-chip was performed according to Agilent Mammalian ChIP-on-chip protocol. Putative Mlip targets were analyzed using the UCSC Cis-Genome Browser database and DAVID (Database for Annotation, Visualization and Integrated Discovery, 2008) bioinformatics tool for analysis of molecular function ontologies. Gene-specific ChIP-PCR was performed with the following primers RARA (5'-TTC TTT CCC CCT ATG CTG GGT-3'; $5^{\prime}$-GGG AGG GCT GGG TAC TAT CTC-3'), SIX1 (5'-ATG CTG CCG TCG TTT GGT T-3'; $5^{\prime}$-CCT TGA GCA CGC TCT CGT T- $\left.3^{\prime}\right)$, SIX3 (5'-CCG GAA GAG TTG TCC ATG TTC-3'; 5'-CGA CTC GTG TTT GTT GAT GGC-3'), SIX4 (5'-CCA CGG TTT TTC CCT GAC CC-3'; $5^{\prime}$-GGT TGC ATA GTT AGT GTT GCT GA-3') and MyoG (5'-GCG GAC TGA GCT CAG CTT AAG-3'; 5'-GCT GTC CAC GAT GGA CGT AAG-3').

\subsection{Nuclear Extracts and Electrophoretic Mobility Shift Assays (EMSA)}

Nuclear protein extracts from cultured C2C12 myoblasts were prepared essentially as described by Farrance and Ordahl [14]. DNA-protein binding was assayed with a double stranded DNA oligonucleotide probe that was biotin 3'end DNA labeled (Pierce) or ${ }^{32}$ P-labelled. EMSA were performed as described by Ueyama et al. [15]. Reactions were performed in binding buffer (20 mM HEPES ( $\mathrm{pH} 7.9$ ), 10\% glycerol, $50 \mathrm{mM} \mathrm{KCl}$, $0.05 \%$ NP-40, $0.5 \mathrm{mM}$ EDTA, $0.5 \mathrm{mM}$ DTT, and $1 \mathrm{mM}$ PMSF) in the presence of $0.5 \mu \mathrm{g}$ of Poly (dI-dC), a nonspecific competitor; Mlip protein generated by in vitro coupled transcription-translation using the TNT Coupled T7/Sp6 Wheat Germ extract system (Promega, Madison, WI, USA) for $20 \mathrm{~min}$ at room temperature. Products of the binding reactions were resolved by $6 \%$ polyacrylamide gel electrophoresis (PAGE) $0.5 \mathrm{X}$ TBE gel 
for 3-4 h at $10 \mathrm{mM}$. Biotin labeled probe binding reactions were transferred to a nylon membrane (Millipore, Burlington, MA, USA) and biotin-labeled DNA was detected by chemiluminescence (Promega). Then 32 P-labelled probe gels were dried and analyzed with a phosphorimager (Amersham). DNA oligonucleotide probe sequence for EMSA, the sequence for Six3 (5'-GCA GGA TCC CTA CCC CAA CCC CAG CAA GAA ACG C-3'; 5'-GCG TTT CTT GCT GGG GTT GGG GTA GGG ATC CTG C-3').

\subsection{Reporter Assay}

Luciferase reporter assays were performed with the Mammalian Two-Hybrid Assay Kit (Agilent Technologies). Mlip was subcloned in frame with a GAL4-DBD of the pCMV$\mathrm{BD}$ vector and subsequently co-transfected into HEK293 cells with a pFR-Luc reporter plasmid that contained the GAL4 promotor driven Luciferase using Lipofectamine reagent (Invitrogen). Twenty-four h post-transfection lysates were harvested with a passive lysis buffer (Promega) and luciferase activity was determined by a Dual-Luciferase reporter assay system (Promega) as per the manufacturer's protocol.

\subsection{Transfection and Generation of Stable Mlip Knockdown C2C12 Cell Lines}

Two mouse Mlip-shRNAmir sequences in the pGIPZ vector were purchased from Open Biosystems and non-silencing shRNAmir Control vector (Dharmacon RHS4346). Sequences included: V2LMM-214053 mature-sense: CCA ACT ACT TGC TAA ACT T (mm39 Chr9: 77009596-77009616) and V2LMM-211301 mature-sense: CCT ATA ATG CCT TCT ATT A (mm39 Chr9: 77009435-77009454). C2C12 cells in $60 \mathrm{~mm}$ plates were transfected with $4.0 \mu \mathrm{g}$ of shRNAmir plasmid DNA or non-silencing shRNAmir Control vector using Arrest-In transfection (Open Biosystems) reagent in serum-free, antibiotic-free media. Control cells were generated using a validated non-silencing negative control expressed in the pGIPZ vector. Stable cell lines were established using puromycin $(1.5 \mu \mathrm{g} / \mathrm{mL})$ resistance selection.

\section{Results}

\subsection{Mlip Interacts with Chromatin in Areas of Close Proximity to Developmental Genes}

We recently reported the discovery of Mlip through its interaction with lamin A/C [5]. In addition to interacting with lamin $\mathrm{A} / \mathrm{C}$, we also demonstrated that Mlip co-localizes with the promyelocytic leukemia protein (PML) a tumor suppressor protein. Previous studies demonstrated that lamin A/C and many of the proteins within PML bodies formed microdomains of chromatin organization and transcription [16,17]. Mlip also localizes to micro-domains in the nucleus in close proximity to chromatin, in undifferentiated mouse $\mathrm{C} 2 \mathrm{C} 12$ myoblasts (Figure 1A). To address if Mlip also interacts with chromatin, we performed chromatin immunoprecipitation (ChIP) assays. Since the heart expresses the largest number of Mlip splice forms, we first determined that our Mlip antibody was effective in immunoprecipitating endogenous Mlip from total heart lysates (Figure 1B). We then determined that chromatin was immunoprecipitated through Mlip, in four independent ChIP experiments, using the Mlip specific antibody against chemically crossed-linked Mlip of undifferentiated C2C12 myoblasts. A smear of Mlip-bound DNA (Figure 1C) was observed.

To gain insight into the DNA sequence immunoprecipitated through Mlip, the Mlip enriched chromatin was initially TA-cloned [18], followed by direct-PCR of clones and subsequent sequencing (Figure 1D). Ontological analysis of the genes in close proximity to the Mlip-ChIP fragments was found to be primarily associated with commitment, differentiation, apoptosis and growth (Table 1). With this data, we identified that mouse chromosome $2(182 \mathrm{Mbp})$ and 11 (122 Mbp) enrich for similar gene ontologies. 
A

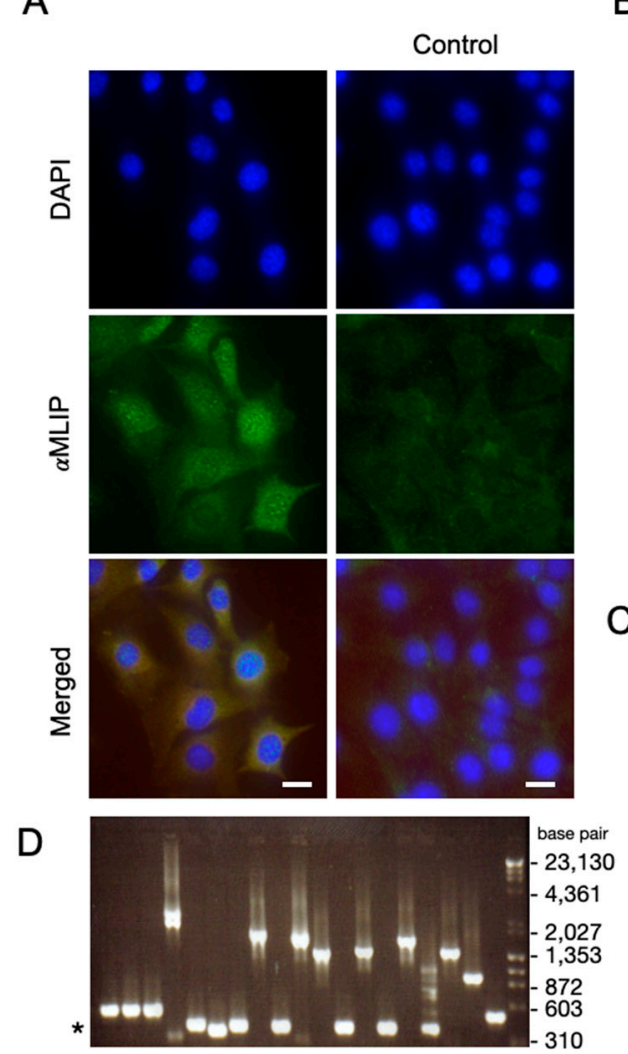

B

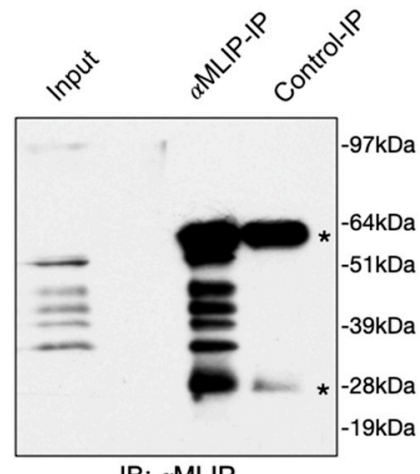

IB: $\alpha$ MLIP

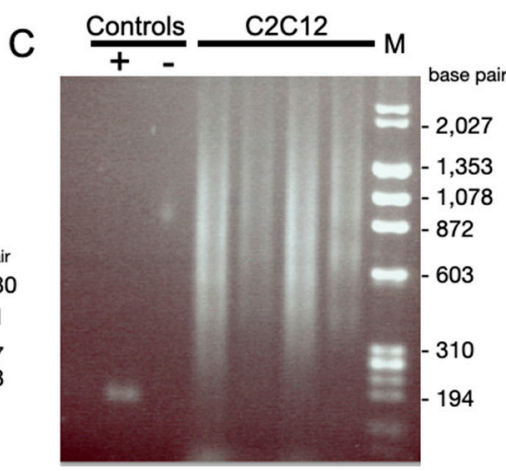

Figure 1. Mlip interacts with genomic DNA. (A) Mlip (green) is localized to the nucleus of C2C12 cell as discrete punctae. Chromatin is stained with DAPI (blue). Control: Mlip-shRNAmir mediated knockdown of endogenouse Mlip mRNA. 20× magnification; Scale bar $=10 \mu \mathrm{M}$ (B) Mlip immunoprecipitations from HL1 whole cell lysates. The control lane used Mlip-peptide neutralized Mlip antibody. "** IgG heavy and light chains. (C) Chromatin immunoprecipitation (ChIP) was performed with an Mlip specific antibody after chemical cross-linking. Mlip-ChIP enriched DNA (4 independent replicates) were isolated and resolved on an agarose gel and subsequently TA-cloned into the pCR-II plasmid and transformed into bacteria. Positive control (+), chromatin was immunoprecipitated with an anti-Histone 3 antibody; subsequently the immunoprecipitated chromatin was PCR amplified with primers targeting a known Histone 3 binding site. Negative control (-), normal rabbit pre-immune sera was used as an IgG negative control for the ChIP. (D) The MLIP mediated ChIP DNA was TA-cloned into pCRII. Direct PCR was performed off pCRII-MLIP-ChIP-DNA transformed colonies with primers targeting plasmid flanking regions of the MLIP-ChIP enriched DNA and visualized on an agarose gel. PCR product sequences were identified by DNA sequencing. "**" size of no insert cloned.

To further identify Mlip chromatin targets, we designed DNA arrays that tiled out the smaller mouse chromosome 11 (1690 genes, Supplementary Table S1), thereby allowing for an additional 1600 promoter regions $(-5 \mathrm{kbp}$ to $+2 \mathrm{kbp}$ around the transcriptional start sites) of genes on the remaining mouse chromosomes (Supplementary Table S2) with similar ontologies as those observed in our preliminary studies (Table 1) to be tiled out on the DNA arrays. The Mlip ChIP-enriched DNA was labeled and hybridized to our custom designed Agilent DNA microarrays (ChIP-on-Chip). The results from two independent biological replicates identified 133 putative Mlip chromatin targets (Supplementary Table S1). 
Table 1. Ontological analysis of cloned Mlip mediated chromatin immunoprecipitated DNA fragments.

\begin{tabular}{|c|c|c|c|c|c|c|}
\hline Gene. & Differentiation & Commitment & Apoptosis & Proliferation & Survival & Growth \\
\hline Akt2 & $\mathbf{x}$ & & $\mathbf{x}$ & $\mathbf{x}$ & $\mathbf{x}$ & $x$ \\
\hline CREM & & & & $x$ & & \\
\hline SOX5 & & $x$ & $\mathbf{x}$ & & & \\
\hline \multicolumn{7}{|l|}{ PEL1 } \\
\hline KIF5C & & & & & & $\mathbf{x}$ \\
\hline PLC1 & & & & $\mathbf{x}$ & $\mathbf{x}$ & $\mathbf{x}$ \\
\hline Met & & & & $\mathbf{x}$ & $\mathbf{x}$ & $\mathbf{x}$ \\
\hline MMP3 & $\mathbf{x}$ & & & $\mathbf{x}$ & $\mathbf{x}$ & \\
\hline Runx & $x$ & & $\mathbf{x}$ & $x$ & & $\mathbf{x}$ \\
\hline \multicolumn{7}{|l|}{ Nek7 } \\
\hline FLI1 & $\mathbf{x}$ & & $\mathbf{x}$ & $\mathbf{x}$ & & $\mathbf{x}$ \\
\hline PP2R3A & & & & & $\mathbf{x}$ & \\
\hline Notch2 & $\mathbf{x}$ & $\mathbf{x}$ & $\mathbf{x}$ & $\mathbf{x}$ & $\mathbf{x}$ & $\mathbf{x}$ \\
\hline
\end{tabular}

To minimize bias, since only mouse chromosome 11 was tiled on the DNA array, analysis of the ontological enrichment of the 45 putative Mlip gene targets identified on chromosome 11 (Supplementary Table S1) by DAVID Bioinformatics Resources [19,20] revealed a significant enrichment of genes that are involved in multicellular organismal development $(p<0.000044)$ and developmental process $(p<0.001)$ (Table 2$)$ as compared to all chromosome 11 encoded genes. Gene-specific ChIP-PCR was performed on a selected number of identified Mlip targets to verify our ChIP-on-Chip data (Figure 2A). We observed myogenin (MyoG), retinoic acid receptor alpha (RAR $\alpha$ ) and Sine oculis homeobox homolog 1/4 (Six1/4) in the Mlip mediated immunoprecipitated chromatin (Figure 2A).

Table 2. Biological processes enriched for on mouse chromosome 11 by Mlip mediated chromatin immunoprecipitation.

\begin{tabular}{lcc}
\hline & $\begin{array}{c}\text { Mlip } \text { Enriched } \\
\text { Chr 11 Genes }\end{array}$ & All Chr 11 Genes \\
\hline \multicolumn{1}{c}{ Gene Ontology Term (Biological Process) } & $p$-Value & $p$-Value \\
\hline 0007275 multicellular organismal development & 0.00004415 & 0.01820829 \\
0032502 developmental process & 0.00099698 & 0.03170131 \\
0009987 cellular process & 0.00146013 & 0.00000553 \\
0016043 cellular component organization \& biogenesis & 0.00175770 & 0.00000001 \\
0048731 system development & 0.00243918 & 0.04057584 \\
0006665 sphingolipid metabolic process & 0.00257593 & ND \\
0048856 anatomical structure development & 0.00297968 & 0.02503862 \\
0006886 intracellular protein transport & 0.00359201 & 0.00028535 \\
0048513 organ development & 0.00386375 & ND \\
0043407 negative regulation of MAP kinase activity & 0.00476669 & 0.03816350 \\
0009888 tissue development & 0.00621520 & ND \\
0006629 lipid metabolic process & 0.00952032 & ND \\
\hline
\end{tabular}

We performed Electrophoretic Mobility Shift Assays (EMSA) on targets identified by the ChIP-on-Chip assay (Supplemental Table S1) to substantiate these results. A Gel SuperShift Assay with our Mlip specific antibody showed that Mlip is part of the Six3 shifted complex (Figure 2B). This super-shifted complex was disrupted by specific competition with unlabeled Six3 competitors as well as with a peptide neutralized Mlip antibody. Results demonstrated that the mobility shift was specifically due to Mlip's association with the DNA-protein complex (Figure 2B). To determine if the binding of Mlip with the SIX3 probe was direct, Mlip was translated in vitro using a cell-free, wheat-germ-based, transcription-coupled translation system. The biotin-labeled SIX3 probe was incubated with the in vitro translated Mlip. We observed a direct interaction between Mlip and the Six3 sequence (Figure 2C). A 50-molar excess of unlabeled Six3 probe competed out the labeled Six3 probe. This strongly suggests that Mlip is able to interact directly with DNA. 
A

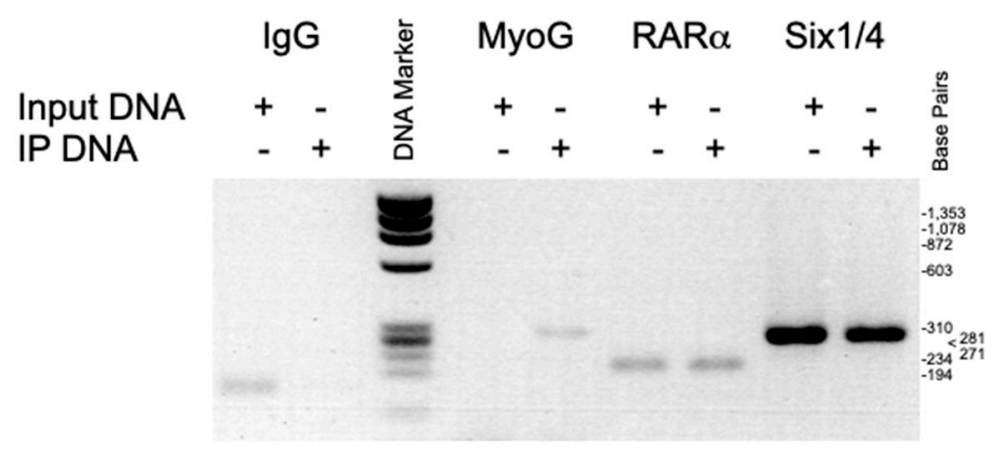

C

B

$\begin{array}{rccccc}\text { Pre-immune } & - & - & - & - & + \\ \text { MLIP Antibody } & - & - & - & + & - \\ \mathrm{C}_{2} \mathrm{C}_{12} \mathrm{NE} & - & + & + & + & + \\ \text { Six3 Competitor } & - & + & - & - & - \\ \text { Six3 Probe } & + & + & + & + & +\end{array}$

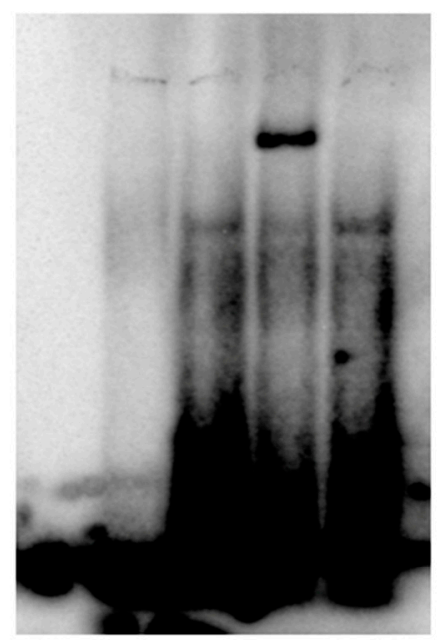

Text $\mathrm{D}$

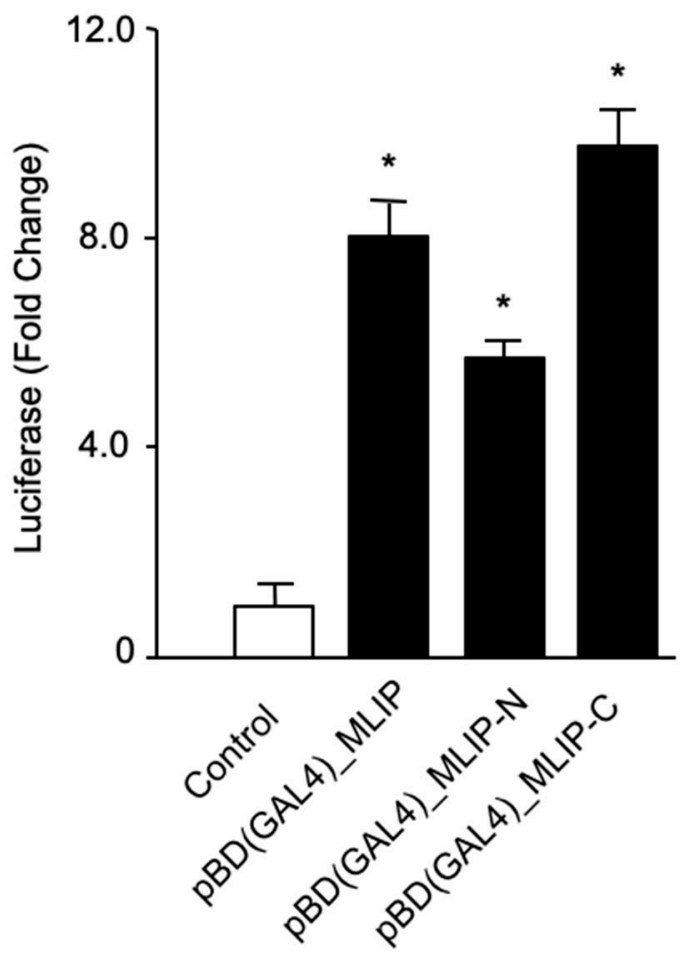

Figure 2. Mlip binds DNA and activates transcription. (A) Gene-specific PCR of Mlip genomic DNA targets. (B) Labeled SIX3 probe was mixed with 2 ug of $\mathrm{C} 2 \mathrm{C} 12$ nuclear extracts (NE) and analyzed via gel shift assays. The complex was super-shifted by the addition of an Mlip specific polyclonal antibody. Mlip peptide neutralized antibody was unable to super-shift the SIX3 associated complex. (C) In vitro translated Mlip was tested for its ability to interact with labeled SIX3 probe by gel shift assay. The labeled SIX3 probe being competed away with a 50-fold molar excess of unlabeled SIX3 probe. (D) Mlip full-length, N-terminal (exons 1-3) and C-terminal Mlip (exons 5-12) fused to a GAL4 DNA binding domain trans-activates a GAL4-Luciferase promoter in HEK293 cells. The assay was repeated 3 times, ${ }^{*}=p<0.01$ versus control, pBD-p53 (Agilent). ' + ' = addition of reagent. '-' symbol means 'no reagent added'. 


\subsection{Mlip Contains a Transcriptional Activation Domain}

To determine if Mlip contains a transcriptional activation domain, we performed co-transfection experiments with HEK293 cells using a GAL4 promoter-luciferase reporter construct together with an expression vector in which the Gal4-DNA-bindingdomain was fused to Mlip (GAL4-DBD-Mlip). We found that GAL4-DBD-Mlip significantly $(p<0.01)$ trans-activated the luciferase reporter through the GAL4 promoter by $\sim 8$-fold over the control in HEK293 cells (Figure 2D). Expression of the N-terminal half of Mlip (exons $1-3)$ fused to a GAL4-DBD (GAL4-DBD-Mlip-N) resulted in a significant $(35 \%, p<0.05)$ reduction in the transcriptional activity of Mlip, whereas the expression of the C-terminal half of Mlip (exons 5-12, GAL4-DBD-Mlip-C) led to a significant (25\%) increase in Mlip's transcriptional activity (Figure 2D). Mlip's intrinsic transcriptional activity is associated with both the N- and C-terminal regions of Mlip, as both activate transcription, with the C-terminal region of Mlip being the more potent of the two within the context of GAL4 fusion (Figure 2D). The C-terminal region of Mlip is comprised of the highly conserved Mlip domain that encompasses exons 8 to 12 [5]. Taken together, our data strongly supports the view that Mlip may function as a transcription regulator since Mlip is found in a complex with chromatin and is able to stimulate DNA transcription.

\subsection{Impaired Myotube Formation in MLIP-Depleted C2C12 Myoblasts}

To verify the biological significance of Mlip's interaction with chromatin of C2C12 myoblasts, Mlip was specifically and stably knocked down in C2C12 cells utilizing shRNAmir technology [21,22]. Initiating differentiation of C2C12 myoblasts by the withdrawal of serum led to the appearance of two additional splice forms of Mlip (30 kDa and $45 \mathrm{kDa}$ ) within 0.25 days with no observed changes in the expression of the $25 \mathrm{kDa}$ Mlip splice variant (data not shown). Total Mlip protein expression peaked at 2 days of differentiation and all three Mlip forms persisted throughout differentiation of the $\mathrm{C} 2 \mathrm{C} 12$ myotubes (Figure 3, top left panel). The distribution of Mlip cellular localization during C2C12 differentiation was assessed by indirect immunofluorescence (Supplemental Figure S1). In undifferentiated $\mathrm{C} 2 \mathrm{C} 12$ myoblast ( 0 days) we observed punctae nuclear staining that persists at both days 1 and 2 differentiation timepoints. Myofibres (days 2 to 5) have reduced Mlip puntate in the nucleus with increased cytoplasmic staining (Supplemental Figure S1). Taken together, these observations of a change in Mlip isoform expression patterns and cellular distribution suggest Mlip may have alternative functions in myoblasts when compared to myofibres.

Two independent Mlip-knockdown (KD) lines in C2C12 were established (Figure 3, top panel); KD1 and KD2 using shRNAmir's targeted to the 3'-UTR of Mlip. We were able to suppress Mlip protein by $>95 \%$ in two independent, undifferentiated C2C12 myoblast Mlip-knockdown lines Mlip-KD1 and Mlip-KD2; (Figure 3, top panel). Morphologically these stable, Mlip knocked down undifferentiated C2C12, myoblasts appeared normal. We observed no difference in the rate of cellular proliferation (doubling time) between controls $(10.6 \pm 1.4 \mathrm{~h})$ and stably knockdown Mlip cells $(10.3 \pm 0.83 \mathrm{~h}, n=3, p=0.77)$ and these were similar to previously reported C2C12 doubling times of $12 \mathrm{~h}$ [23].

Upon differentiation of Mlip-KD1 and Mlip-KD2, we observed a small increase in Mlip expression in both knockdown lines (Figure 3) when compared to the undifferentiated myoblast Mlip KD lines (Figure 3, top panels). There was a significant reduction in the expression of the myogenic regulatory factors $\mathrm{MyoD}$ and $\mathrm{MyoG}$, as compared to the parental cell line (Figure 3). The decrease in MyoD and MyoG expression was accompanied by a discernible reduction of myosin heavy chain expression in both Mlip knockdown lines (Figure 4A) with few multinucleated myotubes observed after 5 days of differentiation (Figure 4A, bottom row). The fusion index, the proportion of total nuclei inside myotubes, was determined after 3 days of differentiation and showed a significant $(p<0.001$, $n=3)$ decrease in myoblast fusion between the control (35.1 $\pm 4 \%$ ) and both Mlip-KD1 $(2.76 \pm 0.52 \%)$ and Mlip KD2 (10.0 $\pm 1.35 \%)$ (Figure 4B). 


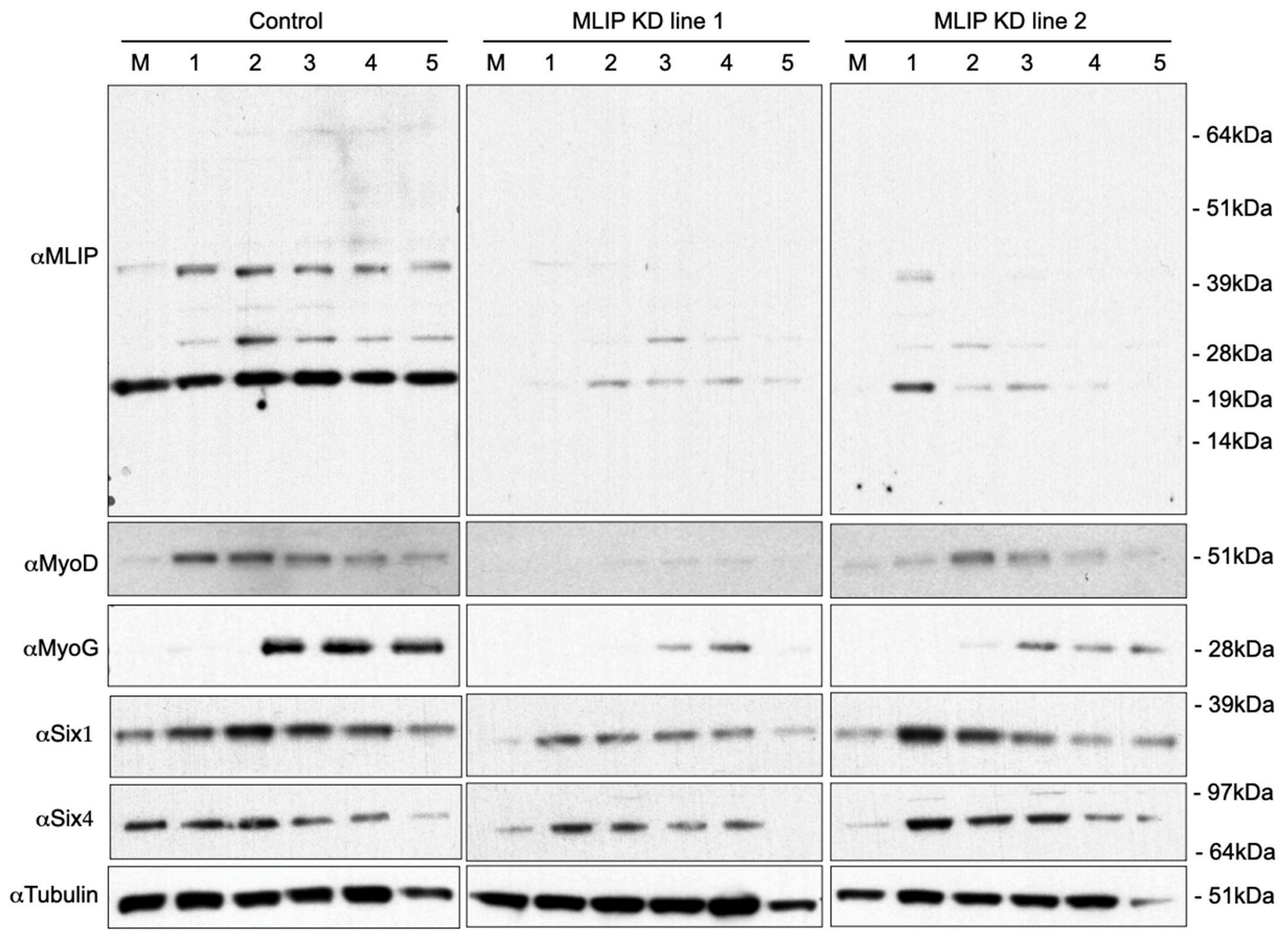

Figure 3. Mlip is necessary for $\mathrm{C} 2 \mathrm{C} 12$ myoblast differentiation into myotubes. Western Blot analysis of $\mathrm{C} 2 \mathrm{C} 12$ myoblasts (M) and C2C12 cells grown in differentiation media was performed using anti-Mlip, anti-MyoD, anti-MyoG, anti-Six1 and anti-SIX4 polyclonal antibodies. Lysates were isolated every day for 5 days of differentiation. Control cells were generated using a validated non-silencing negative control.

The Mlip ChIP-on-Chip experiments (Supplemental Table S1) identified several members of the Six homeobox gene family (Six1, Six2, Six3, Six4, and Six5) as potential targets of Mlip. The Six family of transcription factors, specifically Six1 and Six4, are essential for skeletal muscle development and for myoblast differentiation [13,24-27]. The knockdown of Mlip resulted in a significant decrease in Six1/4 expression in the undifferentiated myoblasts (Figure 3). In contrast, differentiation of the Mlip knockdown lines resulted in a restoration of Six1 and Six4 protein levels in a fashion similar to the control cells (Figure 3). Despite the reestablishment of Six1 and Six4 levels during differentiation, both $\mathrm{MyoD}$ and MyoG expression were markedly reduced and the cells lacked the ability to form normal myotubes (Figure 4). Collectively our data demonstrate that Mlip is required for C2C12 myoblast differentiation into myotubes and that Mlip may exert this role as a transcriptional regulator of the myogenic program. 
A

Control MLIP KD line 1 MLIP KD line 2

$1 d$
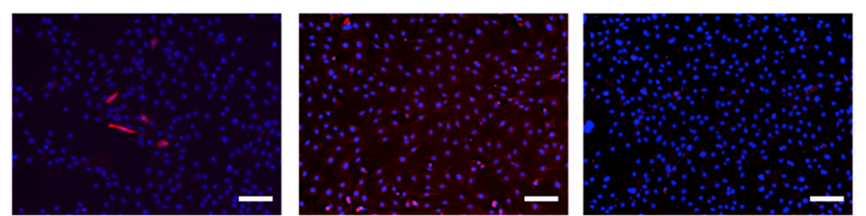

$3 d$
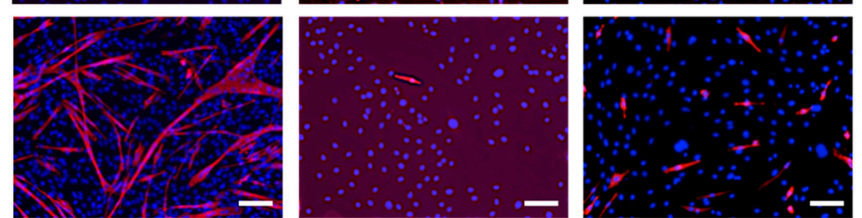

$5 d$
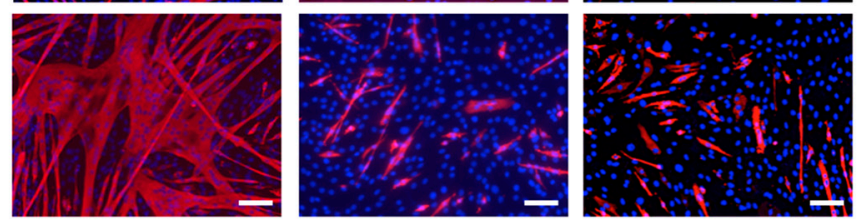

B

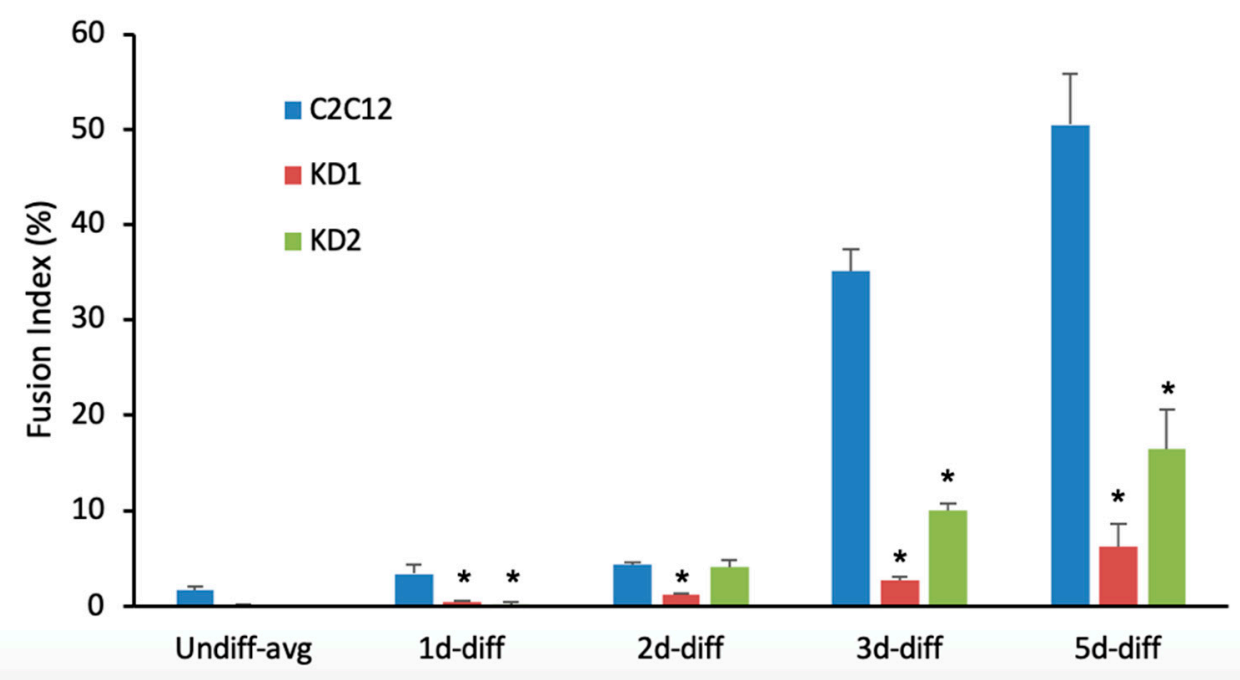

Figure 4. Mlip is required for normal myotube fusion and formation. (A) Indirect immunofluorescence of myosin heavy chain expression during $\mathrm{C}_{2} \mathrm{C}_{12}$ differentiation in the normal or in MLIP-depleted $\mathrm{C} 2 \mathrm{C} 12$ myoblasts. Nuclei were stained with DAPI. $10 \mathrm{x}$ magnification; Scale bar $=50 \mathrm{uM}$ (B) Fusion index calculated as the fraction of total nuclei present inside myotubes (MyHC positive cells) after 0, 1, 2, 3 and 5 days of differentiation. The data represent the pooling of 3 independent differentiations with ${ }^{*}=p<0.001$ vs. control. Control cells were generated using a validated non-silencing negative control.

\section{Discussion}

Mlip was originally discovered through its direct interaction with A-type lamins and is expressed in many different tissues but was found to be most abundant in striated muscle [5]. A large proportion of the mutations described in laminopathies translate into striated muscular dystrophies. We, therefore, used mouse C2C12 myoblasts derived from satellite cells, an established in vitro myoblast model system [28-30], to investigate the role of Mlip in muscle differentiation and its underlying molecular mechanism. Here we demonstrate that Mlip may function as a transcriptional co-factor that targets the regulation of genes involved in cell fate during development, specifically in muscle. We further establish that Mlip is required for muscle differentiation and may regulate the myogenic program through its association with chromatin. 
Previous studies have reported that A-type lamins interact with and organize chromatin [31] that may be through a lamin-histone interaction [32]. Mlip is localized to both the nuclear envelope as well as within nuclear bodies, suggesting to us that Mlip may also interact with chromatin. Using Mlip mediated chromatin immunoprecipitation microarrays, we discovered that Mlip interacts with chromatin in close proximity to genes that encode a variety of transcription factors, many of which have functions that are critical to development and growth of skeletal muscle (PAX3, SIX1, SIX4, JUNB, and MEF2C). A-type lamins have been previously shown to interact directly with transcription factors such as pRB [33,34] SREBP-1 [35] and MOK2 [36], implying that A-type lamins could act as accessory proteins for a subset of tissue determining factors. Our data show that Mlip, with its tissue-specific splicing, may act as a modifier, thus providing a rationale for the phenotypic heterogeneity observed in laminopathies. Furthermore, the recent report by Huang et al. [9] demonstrated that Mlip also interacts with Islet1 (Isl1), an essential transcription factor for cardiac progenitor cell specification, and represses the transcriptional activity of Isl1 [37]. This raises many new and intriguing questions as to what the functional role of Mlip may be, especially since A-type lamins are expressed during cells/tissues commitment [38]. Both Isl1 and A-type lamins play critical roles in the regulation of gene expression and Mlip, through its interaction with both Isl1 and A-type lamins, may play a regulatory role for both Isl1 and A-type lamin function.

Duchenne muscular dystrophy (DMD) is characterized by progressive muscle-wasting disease that affects striated muscles including the limb muscles, the diaphragm, and the heart [39] - all tissues in which Mlip is expressed. Patients afflicted with DMD are diagnosed early in childhood with death usually occurring in the teenage years or early 20 s by cardiorespiratory failure. Like the laminopathies, the underlying molecular pathways leading to DMD are poorly understood even though the genetic mutations (not associated with LMNA) are well characterized, and the histological pathology is well documented. Gene expression profiling of quadricep skeletal muscle biopsies from DMD patients and unaffected control patients were examined [40] and revealed a significant $(p<0.0001$, $n=11$; NCBI GEO profile GDS610 record) reduction in Mlip expression in the DMD affected patients while lamin A/C remained unaffected, suggesting that Mlip protein levels do not affect lamin A/C level of expression, whereas, shRNA mediated down-regulation of lamin A/C expression results in the up-regulation and cellular mislocalization of Mlip [5]. We previously showed that Mlip is localized to both the cytosol and nucleus of C2C12 myoblasts and is found within PML bodies that we believe is indicative of alternative functions beyond binding A-type lamins [5]. Since Mlip interacts with chromatin, then the shuttling of Mlip between these nuclear bodies, the nuclear envelope and cytosol, may be an important regulatory process that may be independent of its association with lamin A/C. Furthermore, this shuttling may be necessary for the coordinated withdrawal of myoblasts from the cell cycle and their fusion to form myotubes.

Not only does Mlip complex with chromatin, it also may function as a transcriptional regulator since Mlip is able to activate transcription. Moreover, this study reveals enrichment of putative Mlip targets with known involvement in a wide variety of developmental processes associated with the organogenesis of striated muscle and brain, suggesting an important role for Mlip in organ development. The reduction or absence of Mlip may have a non-specific global effect on the transcription of many genes, some of which may play an important role in muscle differentiation and function. Mlip may affect specific gene targets important for maintaining myogenic determination of skeletal muscle cells. This observation may have significance in the tissue-specific Mlip isoform distribution that was previously reported [5] in that these Mlip isoform patterns may dictate distinct transcriptional programs required for cell fate during development. This, in turn, may explain the phenotypic heterogeneity observed in laminopathy patients where over 400 mutations in the ubiquitously expressed LMNA gene have been described to date [41-43].

Previous studies have reported a critical role for lamin A/C and emerin in satellite cell differentiation [44-46] with mutations within lamin A of patients being attributed to Emery- 
Dreifuss and limb-girdle muscular dystrophy. The reduction of A-type lamin expression in $\mathrm{C} 2 \mathrm{C} 12$ cells resulted in an up-regulation of Mlip expression and mislocalization of Mlip in the myoblast [5]. Mlip's interaction with A-type lamins in the nuclear envelope may prove to be essential for normal differentiation. MLIP interacts with a domain found in the first 130 amino acids of A-type lamin [5]. Mutations, particularly those in the first 130 amino acids of lamin A/C, may alter the kinetics of the lamin A/C interaction with Mlip and result in reduced myotube formation. In $\mathrm{C} 2 \mathrm{C} 12$ cells we observed the up-regulation of two additional Mlip splice forms as myoblasts differentiate and fuse to form myotubes. Since Mlip binds chromatin and also contains at least one transactivation domain, the loss or reduction of Mlip could affect the expression of numerous genes. The loss of Mlip has a functional consequence in mouse $\mathrm{C} 2 \mathrm{C} 12$ myoblasts in that the temporal activation of both MyoD and MyoG were significantly reduced, which translated into a significant reduction in the fusion of these myoblasts to form myotubes. Because activation of MyoD and MyoG expression and subsequent differentiation was almost abolished in the absence of Mlip, we believe that in $\mathrm{C} 2 \mathrm{C} 12$ myoblasts, Mlip is essential for muscle terminal differentiation and that it exerts its effects at least in part by enabling expression of myogenic regulatory factors. Our findings shed new light on the biological function of Mlip as a potential transcriptional co-factor and the role it may play in skeletal muscle differentiation.

Supplementary Materials: The following are available online at https:/ /www.mdpi.com/2073-4 409/10/3/615/s1, Figure S1: C2C12 differentiation, Table S1: Mlip mediated immunoprecipitated chromatin sequences and proximate genes.

Author Contributions: Conceptualization, E.A. and P.G.B.; methodology, E.A., A.B. and P.G.B.; validation, E.A., A.B. and P.G.B.; formal analysis, A.B. and P.G.B.; writing—original draft preparation, E.A.; writing-review and editing, A.B. and P.G.B.; supervision, A.B. and P.G.B.; project administration, P.G.B.; funding acquisition, P.G.B. All authors have read and agreed to the published version of the manuscript.

Funding: This research was funded by an Operating grant from Canadian Health Research Institute and Heart and Stroke Foundation of Canada to PGB (CIHR grant \#82748 \& 119470; HSFO \#0007) and A.B. (CIHR grant \#119458). Ontario Graduate Student Science and Technology (OGSST) provided a stipend to E.A.

Institutional Review Board Statement: Not applicable.

Informed Consent Statement: Not applicable.

Data Availability Statement: Data sharing is not applicable to this article.

Acknowledgments: We greatly appreciate Balwant Tuana (University of Ottawa) and Alex Stewart (University of Ottawa Heart Institute) for their critical assessment of this manuscript, and Yubing Liu for assistance with the ChIP-on-chip experiments.

Conflicts of Interest: The authors declare no conflict of interest.

\section{References}

1. Broers, J.L.V.; Ramaekers, F.C.S.; Bonne, G.; Yaou, R.B.; Hutchison, C.J. Nuclear lamins: Laminopathies and their role in premature ageing. Physiol. Rev. 2006, 86, 967-1008. [CrossRef]

2. Capell, B.C.; Collins, F.S. Human laminopathies: Nuclei gone genetically awry. Nat. Rev. Genet. 2006, 7, 940-952. [CrossRef]

3. Scaffidi, P.; Misteli, T. Lamin A-dependent nuclear defects in human aging. Science 2006, 312, 1059-1063. [CrossRef]

4. Worman, H.J.; Courvalin, J.-C. How do mutations in lamins A and C cause disease? J. Clin. Invest. 2004, 113, 349-351. [CrossRef] [PubMed]

5. Ahmady, E.; Deeke, S.A.; Rabaa, S.; Kouri, L.; Kenney, L.; Stewart, A.F.R.; Burgon, P.G. Identification of a novel muscle A-type lamin-interacting protein (MLIP). J. Biol. Chem. 2011, 286, 19702-19713. [CrossRef] [PubMed]

6. Cattin, M.-E.; Deeke, S.A.; Dick, S.A.; Verret-Borsos, Z.J.A.; Tennakoon, G.; Gupta, R.; Mak, E.; Roeske, C.L.; Weldrick, J.J.; Megeney, L.A.; et al. Expression of murine muscle-enriched A-type lamin-interacting protein (MLIP) is regulated by tissue-specific alternative transcription start sites. J. Biol. Chem. 2018, 293, 19761-19770. [CrossRef] [PubMed]

7. Santini, M.P.; Forte, E.; Harvey, R.P.; Kovacic, J.C. Developmental origin and lineage plasticity of endogenous cardiac stem cells. Development 2016, 143, 1242-1258. [CrossRef] [PubMed] 
8. Moretti, A.; Lam, J.; Evans, S.M.; Laugwitz, K.-L. Biology of Isl1+ cardiac progenitor cells in development and disease. Cell. Mol. Life Sci. 2007, 64. [CrossRef]

9. Huang, Z.-P.; Young Seok, H.; Zhou, B.; Chen, J.J.-F.; Chen, J.J.-F.; Tao, Y.; Pu, W.T.; Wang, D.-Z. CIP, a cardiac Isl1-interacting protein, represses cardiomyocyte hypertrophy. Circ. Res. 2012, 110, 818-830. [CrossRef] [PubMed]

10. Cattin, M.-E.; Wang, J.; Weldrick, J.J.; Roeske, C.L.; Mak, E.; Thorn, S.L.; DaSilva, J.N.; Wang, Y.; Lusis, A.J.; Burgon, P.G. Deletion of MLIP (Muscle-enriched A-type Lamin-interacting Protein) Leads to Cardiac Hyperactivation of Akt/Mammalian Target of Rapamycin (mTOR) and Impaired Cardiac Adaptation. J. Biol. Chem. 2015, 290, 26699-26714. [CrossRef]

11. Huang, Z.-P.; Kataoka, M.; Chen, J.; Wu, G.; Ding, J.; Nie, M.; Lin, Z.; Liu, J.; Hu, X.; Ma, L.; et al. Cardiomyocyte-enriched protein CIP protects against pathophysiological stresses and regulates cardiac homeostasis. J. Clin. Invest. 2015, 125, $4122-4134$. [CrossRef]

12. Liu, J.; Huang, Z.P.; Nie, M.; Wang, G.; Silva, W.J.; Yang, Q.; Freire, P.P.; Hu, X.; Chen, H.; Deng, Z.; et al. Regulation of myonuclear positioning and muscle function by the skeletal muscle-specific CIP protein. Proc. Natl. Acad. Sci. USA 2020, 117, 19254-19265. [CrossRef]

13. Liu, Y.; Chu, A.; Chakroun, I.; Islam, U.; Blais, A. Cooperation between myogenic regulatory factors and SIX family transcription factors is important for myoblast differentiation. Nucleic Acids Res. 2010, 38, 6857-6871. [CrossRef]

14. Farrance, I.K.; Ordahl, C.P. The role of transcription enhancer factor-1 (TEF-1) related proteins in the formation of M-CAT binding complexes in muscle and non-muscle tissues. J. Biol. Chem. 1996, 271, 8266-8274. [CrossRef] [PubMed]

15. Ueyama, T.; Zhu, C.; Valenzuela, Y.M.; Suzow, J.G.; Stewart, A.F.R. Identification of the Functional Domain in the Transcription Factor RTEF-1 That Mediates 1-Adrenergic Signaling in Hypertrophied Cardiac Myocytes. J. Biol. Chem. 2000, 275, 17476-17480. [CrossRef] [PubMed]

16. Dechat, T.; Pfleghaar, K.; Sengupta, K.; Shimi, T.; Shumaker, D.K.; Solimando, L.; Goldman, R.D. Nuclear lamins: Major factors in the structural organization and function of the nucleus and chromatin. Genes Dev. 2008, 22, 832-853. [CrossRef]

17. Kandert, S.; Wehnert, M.; Müller, C.R.; Buendia, B.; Dabauvalle, M.-C. Impaired nuclear functions lead to increased senescence and inefficient differentiation in human myoblasts with a dominant p.R545C mutation in the LMNA gene. Eur. J. Cell Biol. 2009, 88, 593-608. [CrossRef] [PubMed]

18. Zhou, M.Y.; Gomez-Sanchez, C.E. Universal TA cloning. Curr. Issues Mol. Biol. 2000, 2, 1-7. [PubMed]

19. Dennis, G.; Sherman, B.T.; Hosack, D.A.; Yang, J.; Gao, W.; Lane, H.C.; Lempicki, R.A. DAVID: Database for Annotation, Visualization, and Integrated Discovery. Genome Biol. 2003, 4, P3. [CrossRef] [PubMed]

20. Huang, D.W.; Sherman, B.T.; Lempicki, R.A. Systematic and integrative analysis of large gene lists using DAVID bioinformatics resources. Nat. Protoc. 2008, 4, 44-57. [CrossRef]

21. Rubinson, D.A.; Dillon, C.P.; Kwiatkowski, A.V.; Sievers, C.; Yang, L.; Kopinja, J.; Zhang, M.; McManus, M.T.; Gertler, F.B.; Scott, M.L.; et al. A lentivirus-based system to functionally silence genes in primary mammalian cells, stem cells and transgenic mice by RNA interference. Nat. Genet. 2003, 33. [CrossRef] [PubMed]

22. Kesireddy, V.; Van Der Ven, P.F.M.; Fürst, D.O. Multipurpose modular lentiviral vectors for RNA interference and transgene expression. Mol. Biol. Rep. 2010, 37. [CrossRef] [PubMed]

23. Rossi, F.; Charlton, C.A.; Blau, H.M. Monitoring protein-protein interactions in intact eukaryotic cells by beta-galactosidase complementation. Proc. Natl. Acad. Sci. USA 1997, 94, 8405-8410. [CrossRef] [PubMed]

24. Chakroun, I.; Yang, D.; Girgis, J.; Gunasekharan, A.; Phenix, H.; Kærn, M.; Blais, A. Genome-wide association between Six4, MyoD, and the histone demethylase Utx during myogenesis. FASEB J. 2015, 29, 4738-4755. [CrossRef]

25. Liu, Y.; Chakroun, I.; Yang, D.; Horner, E.; Liang, J.; Aziz, A.; Chu, A.; De Repentigny, Y.; Dilworth, F.J.; Kothary, R.; et al. Six1 regulates MyoD expression in adult muscle progenitor cells. PLoS ONE 2013, 8, e67762. [CrossRef]

26. Giordani, J.; Bajard, L.; Demignon, J.; Daubas, P.; Buckingham, M.; Maire, P. Six proteins regulate the activation of Myf5 expression in embryonic mouse limbs. Proc. Natl. Acad. Sci. USA 2007, 104, 11310-11315. [CrossRef] [PubMed]

27. Grifone, R.; Demignon, J.; Houbron, C.; Souil, E.; Niro, C.; Seller, M.J.; Hamard, G.; Maire, P. Six1 and Six4 homeoproteins are required for Pax3 and Mrf expression during myogenesis in the mouse embryo. Development 2005, 132, 2235-2249. [CrossRef] [PubMed]

28. Blau, H.M.; Chiu, C.P.; Webster, C. Cytoplasmic activation of human nuclear genes in stable heterocaryons. Cell 1983, 32, 1171-1180. [CrossRef]

29. Schneider, M.D.; Olson, E.N. Control of myogenic differentiation by cellular oncogenes. Mol. Neurobiol. 1988, 2, 1-39. [CrossRef]

30. Olson, E.N. Proto-oncogenes in the regulatory circuit for myogenesis. Semin. Cell Biol. 1992, 3, 127-136. [CrossRef]

31. Glass, C.A.; Glass, J.R.; Taniura, H.; Hasel, K.W.; Blevitt, J.M.; Gerace, L. The alpha-helical rod domain of human lamins A and C contains a chromatin binding site. EMBO J. 1993, 12, 4413-4424. [CrossRef] [PubMed]

32. Taniura, H.; Glass, C.; Gerace, L. A chromatin binding site in the tail domain of nuclear lamins that interacts with core histones. J. Cell Biol. 1995, 131, 33-44. [CrossRef]

33. Markiewicz, E.; Dechat, T.; Foisner, R.; Quinlan, R.A.; Hutchison, C.J. Lamin A/C Binding Protein LAP2alpha Is Required for Nuclear Anchorage of Retinoblastoma Protein. Mol. Biol. Cell 2002, 13, 4401-4413. [CrossRef]

34. Ozaki, T.; Saijo, M.; Murakami, K.; Enomoto, H.; Taya, Y.; Sakiyama, S. Complex formation between lamin A and the retinoblastoma gene product: Identification of the domain on lamin A required for its interaction. Oncogene 1994, 9, $2649-2653$. 
35. Lloyd, D.J.; Trembath, R.C.; Shackleton, S. A novel interaction between lamin A and SREBP1: Implications for partial lipodystrophy and other laminopathies. Hum. Mol. Genet. 2002, 11, 769-777. [CrossRef]

36. Dreuillet, C.; Tillit, J.; Kress, M.; Ernoult-Lange, M. In vivo and in vitro interaction between human transcription factor MOK2 and nuclear lamin A/C. Nucleic Acids Res. 2002, 30, 4634-4642. [CrossRef] [PubMed]

37. Laugwitz, K.-L.; Moretti, A.; Lam, J.; Gruber, P.; Chen, Y.; Woodard, S.; Lin, L.-Z.; Cai, C.-L.; Lu, M.M.; Reth, M.; et al. Postnatal isl1+ cardioblasts enter fully differentiated cardiomyocyte lineages. Nature 2005, 433, 647-653. [CrossRef] [PubMed]

38. Röber, R.A.; Weber, K.; Osborn, M. Differential timing of nuclear lamin A/C expression in the various organs of the mouse embryo and the young animal: A developmental study. Development 1989, 105, 365-378. [PubMed]

39. Watkins, S.C.; Culien, M.J. A quantitative study of myonuclear and satellite cell nuclear size in Duchenne's muscular dystrophy, polymyositis and normal human skeletal muscle. Anat. Rec. 1988, 222, 6-11. [CrossRef]

40. Haslett, J.N.; Sanoudou, D.; Kho, A.T.; Han, M.; Bennett, R.R.; Kohane, I.S.; Beggs, A.H.; Kunkel, L.M. Gene expression profiling of Duchenne muscular dystrophy skeletal muscle. Neurogenetics 2003, 4, 163-171. [CrossRef]

41. Decostre, V.; Ben Yaou, R.; Bonne, G. Laminopathies affecting skeletal and cardiac muscles: Clinical and pathophysiological aspects. Acta Myol. 2005, 24, 104-109.

42. Worman, H.J.; Bonne, G. “Laminopathies": A wide spectrum of human diseases. Exp. Cell Res. 2007, 313, 2121-2133. [CrossRef] [PubMed]

43. Bertrand, A.T.; Chikhaoui, K.; Yaou, R.B.; Bonne, G. Clinical and genetic heterogeneity in laminopathies. Biochem. Soc. Trans. 2011, 39, 1687-1692. [CrossRef] [PubMed]

44. Bakay, M.; Wang, Z.; Melcon, G.; Schiltz, L.; Xuan, J.; Zhao, P.; Sartorelli, V.; Seo, J.; Pegoraro, E.; Angelini, C.; et al. Nuclear envelope dystrophies show a transcriptional fingerprint suggesting disruption of Rb-MyoD pathways in muscle regeneration. Brain 2006, 129, 996-1013. [CrossRef] [PubMed]

45. Frock, R.L.; Kudlow, B.A.; Evans, A.M.; Jameson, S.A.; Hauschka, S.D.; Kennedy, B.K. Lamin A/C and emerin are critical for skeletal muscle satellite cell differentiation. Genes Dev. 2006, 20, 486-500. [CrossRef]

46. Melcon, G.; Kozlov, S.; Cutler, D.A.; Sullivan, T.; Hernandez, L.; Zhao, P.; Mitchell, S.; Nader, G.; Bakay, M.; Rottman, J.N.; et al. Loss of emerin at the nuclear envelope disrupts the Rb1/E2F and MyoD pathways during muscle regeneration. Hum. Mol. Genet. 2006, 15, 637-651. [CrossRef] 